\title{
Bruton's Tyrosine Kinase (Btk) Inhibitor Tirabrutinib Prevents the Development of Murine Lupus
}

\author{
Yuko Ariza, Masayuki Murata, Yoshiko Ueda and Toshio Yoshizawa*
}

Discovery Research Laboratories III, Ono Pharmaceutical Co., Ltd., Osaka, Japan

${ }^{*}$ Corresponding author: Toshio Yoshizawa, Discovery Research Laboratories III, Ono Pharmaceutical Co., Ltd., Osaka, Japan, Tel: +81759611151; Email: to.yoshizawa@ono.co.jp

Received Date: January 20, 2019; Accepted Date: January 28, 2019; Published Date: February 18, 2019

Copyright: (C) 2019 Yuko A, et al. This is an open-access article distributed under the terms of the Creative Commons Attribution License, which permits unrestricted use, distribution, and reproduction in any medium, provided the original author and source are credited.

Citation: Yuko A, Masayuki M, Yoskiko U, Toshio Y (2019) Bruton's Tyrosine Kinase (Btk) Inhibitor Tirabrutinib Prevents the Development of Murine Lupus. Eur Exp Biol Vol. 9 No.1:2.

\section{Abstract}

Systemic Lupus Erythematosus (SLE) is a complex and heterogeneous autoimmune disease associated with the over production of high affinity autoantibodies. Overactivity of B-cell responsiveness to immune stimulation and direct activation of circulating FcR bearing cells are sufficient to initiate inflammatory responses, which may be an essential feature of SLE pathogenesis. Here, we examined the potential efficacy of tirabrutinib using NZB/WF1 and MRL/Ipr mice in the model of spontaneous SLE. Tirabrutinib inhibited the production of anti-dsDNA in serum, and the onset of proteinuria resulted in markedly lower in both lupus-prone mice. Furthermore, the treatment with tirabrutinib resulted in $100 \%$ survival, while $70 \%$ survival was observed in untreated mice. Significant reductions in the numbers of total IgG and anti-dsDNAsecreting B-cells were apparent in spleens from tirabrutinib treated mice. Germinal center B-cells and plasma cells were also significantly lower in tirabrutinib treated mice. Our results demonstrate that treatment with tirabrutinib may simultaneously target autoantibody producing and effector cells to prevent the spontaneous disease development in lupus-prone mice. These data suggest that tirabrutinib may provide promising therapeutic benefit in human lupus and related disorders.

Keywords: Systemic lupus erythematosus; Lupus nephritis; Bruton's tyrosine kinase

\section{Introduction}

Systemic Lupus Erythematosus (SLE) is an autoimmune disease that is characterized by autoantibody production and tissue damage caused by immune complex $[1,2]$. Preclinical and clinical data suggest that pathogenic B-cells contribute to SLE pathogenesis by autoantibody production, antigen presentation, and cytokine generation. Pathogenic autoantibodies have been shown to play a central role in the manifestations of SLE and the titres of anti-dsDNA antibodies rise during flares of SLE disease activity, particularly lupus nephritis. Lupus nephritis is a severe manifestation of SLE with significant morbidity and mortality and reported to affect approximately $60 \%$ of SLE patients [3]. Several biological agents targeting B-cells, such as anti-BAFF [4], anti-CD20 mAb [5,6], anti-CD22 mAb $[7,8]$ have been developed at forefront therapy in SLE. Belimumab, anti-BAFF, has been approved by the FDA, however, clinical trials were not designed to evaluate the efficacy for the treatment of lupus nephritis. Therefore, there is a high unmet need in lupus nephritis.

Bruton's tyrosine kinase (Btk) is a Tec-family kinase that is expressed in most hematopoietic cells. Btk plays a crucial role in the development and activation of B-cells through its activation via the $B C R[9,10]$. Btk mutations in humans leads to $X$-linked Agammaglobulinemia (XLA) which is one of the most frequently inherited immunodeficiency diseases, and is characterized by an almost complete arrest of B-cell differentiation at the pre-B cell stage [11]. Tirabrutinib is an irreversible second-generation Btk inhibitor with excellent efficacy and tolerability in $R / R$ B-cell malignancies [12,13]. Tirabrutinib forms a covalent bond with Cys481 of Btk and, irreversibly inhibits the kinase activity of Btk. Besides an increase in the therapeutic options for B-cell lymphoma, Btk inhibition is also expected to develop in inflammatory disease due to its pleiotropic anti-inflammatory effects. BTK plays critical roles in activation mediated by Fc receptor (FcR), Toll-like receptor in myeloid cells. Several Btk inhibitors have been evaluated in animal models of inflammatory disease including murine lupus models [14-19]. In this study, we found that tirabrutinib attenuated the development of murine lupus in NZB/W F1 and MRL/Ipr mice.

\section{Materials and Methods}

\section{Animal used}

Ten or nineteen-week-old of female Slc:NZBWF1 mice and five-week-old of female MRL/Mpj Jms Slc-lpr/lpr mice were used (Japan SLC, Inc.). All mice were allowed free access to pelleted CRF-1 diet (Oriental Yeast Co., Ltd.) and tap water. The present study was conducted in compliance with the "Guidance for Animal Experiments" and the "Standards for Safety Management of Pathogens" established by Ono Pharmaceutical Co., Ltd. and "Guidelines for Animal Experiments" (Nonclinical Research Center, LSI Medience Corporation). 


\section{Reagents}

Tirabrutinib was obtained from Ono Pharmaceutical Co., Ltd. (Osaka, Japan) and chow containing tirabrutinib was obtained from Oriental Yeast Co., Ltd.

\section{Protocol for NZB/W F1 and MRL/Ipr mouse models}

NZB/W F1 mice were randomized into three treatment groups at $28 \mathrm{wk}$ of age and fed a diet containing tirabrutinib [20], $0.012 \%$ (equivalent to $20 \mathrm{mg} / \mathrm{kg} / \mathrm{day}$ ) and $0.0037 \%(6 \mathrm{mg} / \mathrm{kg} /$ day) from 28 to $37 \mathrm{wk}$. In MRL/lpr mice study, mice were randomized to two treatment groups at $6 \mathrm{wk}$ of age and fed a diet containing tirabrutinib from 6 to $30 \mathrm{wk}$.

\section{Proteinuria}

Urine was collected over time by gently compressing the abdomen of each immobilized mouse. Urinaryprotein concentration was determined by the Bradford method using a commercial Coomassie plus protein assay kit (Pierce Chemical Co).

\section{ELISA}

Blood was collected from the tail vein over time using a capillary tube (Hirschmann Laborgeräte $\mathrm{GmbH}$ ) and allowed to stand statically at room temperature for approximately 1 hour prior to isolation of serum by centrifugation at approximately $1,800 \mathrm{~g}$ at $4^{\circ} \mathrm{C}$ for 10 minutes. Each isolated serum sample was stored frozen (allowable range: $\leq-60^{\circ} \mathrm{C}$ ) until use in the analysis of anti-dsDNA antibodies the level of anti-dsDNA antibody in serum was examined by ELISA (Shibayagi Co., Ltd).

\section{Enzyme-linked immunospot (ELISpot) assay}

After each mouse was euthanized, spleen was collected and splenocytes were prepared from the spleen by homogenization and passed through a nylon mesh. ELISpot assay was performed to evaluate anti-dsDNA-secreting B-cells and total Ig-secreting cells. Ninety-six well filter plates (Millipore) were coated with dsDNA (Alpha diagnostic) or total anti-mouse Ig antibody. Splenocytes were plated and kept for 16 hours at $37 \circ \mathrm{C}$. Plates were washed with PBS containing 0.01 vol\% of Tween 20 , then alkaline phosphatase (ALP)-conjugated anti-mouse IgG $A b$ or ALP-conjugated anti-mouse IgM $A b$ was added and incubated for 2 hours at $37^{\circ} \mathrm{C}$. Plates were washed with PBS and BCIP/ Nitroblue tetrazolium chronmogen was added and incubated for 5 minutes at room temperature. Plates were washed with water and dried. Spots were counted using an analyzer (Immunospot 5.0, CTL).

\section{Flow cytometry}

Splenocytes were fixed with Fix buffer and blocked with Fc Block, then stained with Alexa Fluor 488-CD3, PE-T/B ActivationAntigenPE-Cy7-CD95APC-CD45R/B220, PE-CD138PerCP-Cy5.5CD19 and APC-CD45R/B220 (BD Biosciences). Cells were analyzed using Flow cytometer (BD AccuriBD Biosciences). All splenocytes were primarily gated on live lymphocytes based on forward scatter (FCS) and side scatter (SSC). Germinal center B cells were identified as CD95+GL7+cells in CD3-B220+cells. Plasma cells were identified as CD138+B220lo/int cells in CD3cells. Number of germinal center B cells and plasma cells were expressed per $1 \times 10^{6}$ cells of splenocytes.

\section{Histopathologic evaluation}

The right kidneys were collected and fixed in $4 \%$ paraformaldehyde. The kidneys were embedded in paraffin by a conventional method. Two thin sections, $2 \mu \mathrm{m}$ to $4 \mu \mathrm{m}$ in thickness, were cut from each paraffin block, and then stained with hematoxylin and eosin (H.E.). Lesions were scored by an independent observer using a 0-5 score for glomerular damage in section (5: severe, $>75 \%$, 4: marked, 50\%-75\%, 3: moderate, 25\%-50\%, 2: mild, $10 \%-25 \%$, 1: minimally detectable, $<10 \%, 0$ : no readily detectable glomerular injury in sections) and inflammatory foci in section (5: too numerous to count, 4: widespread distribution of inflammatory cells, 3: limited distribution of inflammatory foci, 2: leukocytic infiltrates are primarily composed of dense foci, 1 : up to 25 lymphocytes, 0 : minimal leukocyte infiltration).

\section{Statistics}

SAS 9.1.3 (EXSUS Ver. 7.7.1, SAS Institute Japan Ltd. [CAC EXICARE Corporation]) was used for analysis of animal data. Data are expressed as the mean \pm standard error. All statistical tests were two-sided with a significance level of $5 \%$.

\section{Results}

\section{Effect of tirabrutinib on anti-dsDNA antibodies in NZB/W F1 mice}

To examine the therapeutic effects of tirabrutinib on spontaneous lupus-prone mice, we first monitored the antidsDNA antibodies in mice and determined the initiation of the treatment. The serum concentrations of anti-dsDNA antibodies became significantly increased at $28 \mathrm{wk}$ of age. Since the levels of anti-dsDNA antibodies correlate with disease activity in systemic lupus erythematosus, the mice were randomized into 3 groups to receive control chow, $0.0037 \%$ and $0.012 \%$ tirabrutinib-formulated chow at 28 wk of age. We then examined the effect on the production of pathogenic antibodies (Figure 1a). Tirabrutinib (0.012\%) significantly prevented further increases in anti-dsDNA antibody levels, while control mice showed a two-fold increase at $31 \mathrm{wk}$, then the levels were slowly starting to decrease until the end of the study at $38 \mathrm{wk}$ (Figure 1b).

\section{Tirabrutinib prevents the development of proteinuria and improves the survival rate in NZB/W F1 mice}

To assess whether tirabrutinib can prevent renal damage, we measured the amount of urinary protein in mice and analysed their survival rate every week from 20 to 38 wk of age (Figure 2). Control chow-treated mice showed a prominent increased level of proteinuria starting at $29 \mathrm{wk}$ until the end of the study. In 
contrast, none of the animals treated with $0.012 \%$ tirabrutinib developed severe proteinuria while some elevation was observed in some mice at the $0.0037 \%$ dose (Figure 2a). Because the primary cause of death in mice is renal failure due to glomerulonephritis, the survival rate in mice was associated with the severity of proteinuria. Proteinuria gradually increased over time and only control treated mice died during the experiment (Figure $\mathbf{2 b}$ ). The correlation between observed time of death and the urinary protein levels was defined at $32 \mathrm{wk}$ as concentrations that were more than $5,000 \mu \mathrm{g} / \mathrm{mL}$.

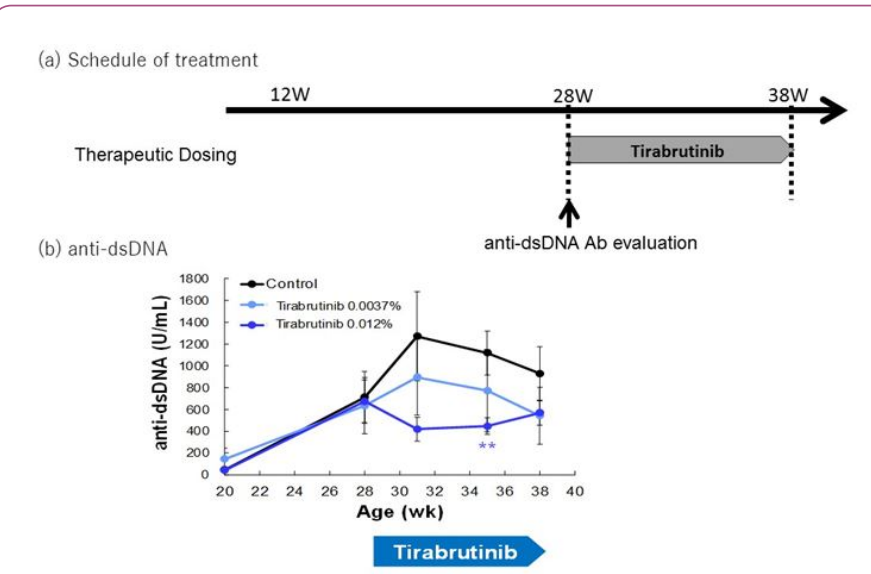

Figure 1: Study design of NZB/WF1 mice, (a) Treatment with tirabrutinib was initiated at $28 \mathrm{wk}$ of age. Mice were randomized into three groups, based on body weight and anti-dsDNA antibody titre. From 28 to 37 wk, mice were allowed free access to diet containing tirabrutinib $(0.012 \%$, equivalent to $20 \mathrm{mg} / \mathrm{kg} /$ day and $0.0037 \%, 6 \mathrm{mg} / \mathrm{kg} /$ day). (b) Serum anti-dsDNA antibody was measured by ELISA. Dunnett's test was used to compare the control and tirabrutinib groups. T test was used to compare the antidsDNA antibody levels at $20 \mathrm{wk}$ and $28 \mathrm{wk}$. A p value of less than $5 \%$ was considered statistically significant. ${ }^{* * *}: p<0.001$, $* *$ : $p<0.01$ vs control group. \#\#\#: $p<0.001$ vs anti-dsDNA antibody level at $20 \mathrm{wk}$.

\section{Significant reduction in antibody-secreting cells, germinal centers and plasma cells}

Btk plays a critical role in the B-cell receptor signaling pathway. In order to clarify the effect of tirabrutinib on B cell development and auto-antibody secretion, we examined the number of splenic antibody-secreting cells by ELISPOT assay (Figure 3). Tirabrutinib significantly reduced the number of splenocytes and splenic anti-dsDNA IgG, anti-dsDNA IgM antibody-secreting cells per splenocytes. Because germinal centers are the primary site at which clonal expansion and affinity maturation of B-cells occur, we next examined the impact of tirabrutinib on the number of germinal center B-cells and plasma cells by flow cytometry. Tirabrutinib significantly reduced the frequency of germinal center B-cells and plasma cells in a dose-dependent manner.
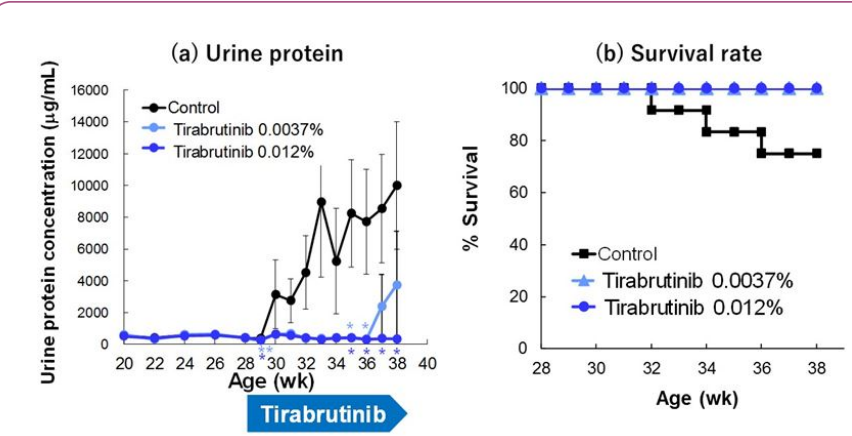

Figure 2: Tirabrutinib prevents progression of proteinuria and improves the survival rate, (a) Urinary protein from 20 to 38 wk of age was determined by Bradford method. (b) Tirabrutinib improves the survival rate in lupus-prone mice. Tirabrutinib treatment was initiated at week 28 , where the animals were fed a diet containing 0.0037 and $0.012 \%$ of tirabrutinib until 38 weeks. Dunnett's test was used to compare the control and tirabrutinib groups. A p value of less than $5 \%$ was considered statistically significant. ${ }^{* *}$ : $p<0.01 *$ : $p<0.05$, vs control group.

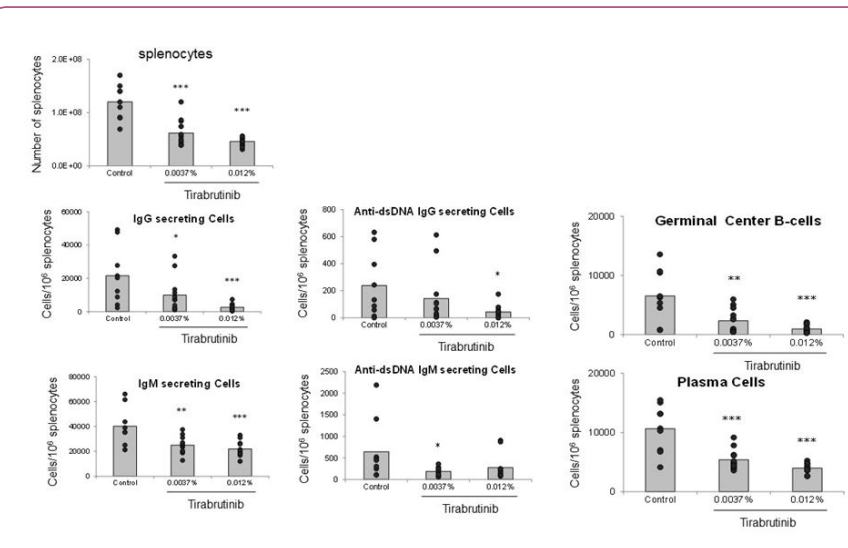

Figure 3: Effect of tirabrutinib on antibody-secreting cells, germinal centers and plasma cells. Splenic, IgG, IgM, antidsDNA IgG and anti-dsDNA IgM secreting cells were determined by ELISpot assay. The germinal center cells were characterized as CD3-, B220+, Fas+,GL7+ cells and plasma cells were characterized as CD3-, B220lo/int, CD138 cells.

\section{Tirabrutinib prevents renal damage and inflammation in NZB/W F1 mice}

To evaluate whether tirabrutinib prevents renal damage and inflammation, tirabrutinib treatment was initiated from $12 \mathrm{wk}$ of age until the subset of mice was sacrificed at $37 \mathrm{wk}$. Upon completion of the study, kidneys were collected and histopathological analysis was conducted. In control treated mice, enlarged glomeruli with variable hypercellularity and increased mesangial matrix were observed (Figure 4a). The histopathology score of control mice was $3.0 \pm 0.4$ for glomerular and $2.3 \pm 0.2$ for inflammation. Tirabrutinib treated mice revealed a significant reduction in glomerular pathology 
and inflammation foci, for $0.0037 \%, 0.4 \pm 0.2$ and $0.1 \pm 0.1$, and for $0.012 \%, 0.9 \pm 0.3$ and $0.4 \pm 0.2$, respectively (Figure $4 \mathrm{~b}$ ). (a) Micrographs images

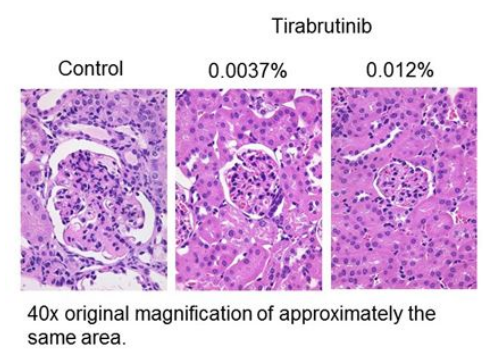

(b) Histopathology score

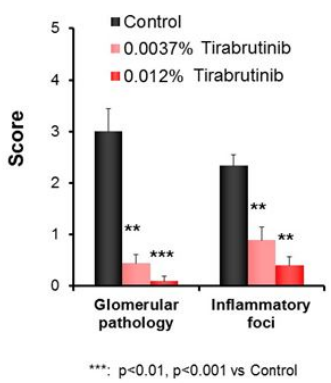

Figure 4: Tirabrutinib prevents nephritis in NZB/W F1 mice. Tirabrutinib was treated from $12 \mathrm{wk}$ of age to $37 \mathrm{wk}$. (a) Micrographs show representative images of renal infiltrates from the treatment of tirabrutinib. (b) Histological evaluation of kidney of tirabrutinib treated mice in glomerular pathology and inflammation foci parameters. 0-5 score for

histopathology were used. Steel test was used to compare the control and tirabrutinib groups. A $p$ value of less than $5 \%$ was considered statistically significant. ${ }^{* * *}: p<0.001,{ }^{* *}: p<0.01$ vs control group.

\section{Tirabrutinib prevents Nephritis in MRL/Ipr Mice}

NZB/W F1 mice has been widely used in studies of lupus pathogenesis and exhibit high titers of autoantibodies and severe nephritis. Another lupus model, MRL/lpr, also exhibit autoantibodies production, enlargement of lymph nodes, and skin lesions. In this model, various immune cell populations, including $B$ and T-cells, contribute to the pathogenesis of the disease. To investigate the effect of tirabrutinib in MRL/lpr mice, treatment was initiated from 6 wk due to the more rapid onset of disease and the multiple manifestations of disease through 30 wk of age. Tirabrutinib dose-dependently suppressed serum anti-dsDNA antibodies (Figure 5a). Control chow-treated mice showed a prominent increased level of proteinuria starting at 23 wk until the end of the study. In contrast, none of the animals treated with tirabrutinib developed severe proteinuria (Figure 5b). Thus, these results support that tirabrutinib may be a promising therapeutic option for lupus nephritis and SLE.

\section{Discussion and Conclusion}

NZB/W F1 and MRL/Ipr models have been extensively used to investigate many drugs [21]. It has been reported that B-celltargeted therapies, such as anti-BAFF or anti-CD20 mAb show efficacy in both models, although rituximab did not meet the clinical endpoints in a human SLE study [22]. Of note, anti-CD20 $\mathrm{mAb}$ treatment in NZB/W F1 mice delayed the onset of the disease without decreasing anti-dsDNA antibodies. Furthermore, prevention of autoantibodies production and renal injury were achieved only after early initiation of the treatment of anti-CD20 mAb [23,24]. Our data show that tirabrutinib alleviates proteinuria with partial inhibition of serum autoantibodies in NZB/W F1 mice. However, both were suppressed completely after early treatment with tirabrutinib in $\mathrm{MRL} / \mathrm{lpr}$ mice. Since long-lived plasma cells which have lost CD20 and Btk expression contribute to the production of autoantibodies, these are not affected by B-cell depletion and tirabrutinib. It is intriguing that NZB/W F1 shows an immunological characteristic of persistent long-lived plasma cells. Together, our data are consistent with those of anti-CD20 $\mathrm{mAb}$ lupus studies, which demonstrated that tirabrutinib is targeting B-cells in murine lupus. (a) Serum anti-dsDNA

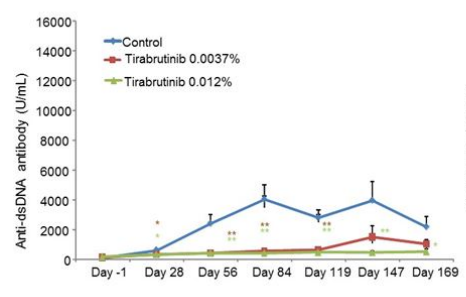

(b) Urine protein

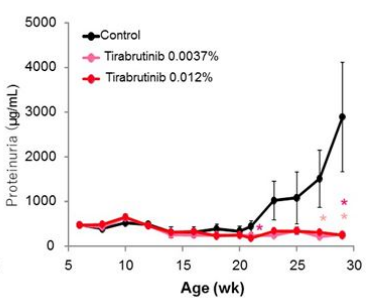

Figure 5: Tirabrutinib prevents progression of proteinurea in $\mathrm{MRL} / \mathrm{Ipr}$ mice. Treatment with tirabrutinib was initiated at 6 wk of age. Mice were randomized into three groups, based on body weight and anti-dsDNA antibody titer. From 6 to $30 \mathrm{wk}$, mice were allowed free access to diet containing tirabrutinib $(0.012 \%$, equivalent to $20 \mathrm{mg} / \mathrm{kg} /$ day and $0.0037 \%, 6 \mathrm{mg} / \mathrm{kg} /$ day). (a) Serum anti-dsDNA antibody was measured by ELISA. (b) Urinary protein from 6 to $30 \mathrm{wk}$ of age was determined by Bradford method. Dunnett's test was used to compare the control and tirabrutinib groups. A p value of less than $5 \%$ was considered statistically significant. ${ }^{* *}$ : $p<0.01, *$ : $p<0.05$, vs control group.

Germinal centers are central to the development of long-lived plasma B cells and their clonal expansion to produce autoantibodies. Disease progression of lupus nephritis is associated with an increase in the number and size of germinal centers. Tfh cells are essential for formation of germinal centers and have been reported to contribute to lupus pathogenesis $[25,26]$. Our data show that tirabrutinib reduced the number of splenic anti-dsDNA antibody producing cells in NZB/W F1 mice. In addition, tirabrutinib reduced the frequency of splenic $\mathrm{GC} B$ cells and plasma cells.

We evaluated the effect of tirabrutinib on renal injury of NZB/W F1 mice. Tirabrutinib showed significant suppression of renal infiltration of inflammatory cells in a dose dependent manner. Since leukocyte infiltration features in both human and experimental lupus nephritis are associated with a progressive loss of renal function, tirabrutinib may affect not only B-cells but others which have Btk expression, such as neutrophils and mononuclear phagocytes. Our previous studies have confirmed that tirabrutinib inhibits immune-receptor signaling in multiple cells through Btk inhibition. Therefore, the anti-inflammatory activity of tirabrutinib on myeloid cells may contribute to the suppression of the lupus nephritis. 
In conclusion, this study demonstrates that tirabrutinib exhibits potential therapeutic value for the treatment of lupus nephritis.

\section{Acknowledgement}

We would like to thank the LSI Medience Corporation for their support in lupus experiments.

\section{References}

1. Nashi E, Wang Y, Diamond B (2010) The role of b cells in lupus pathogenesis. Int J Biochem Cell Biol 42: 543-550.

2. Hostmann A, Jacobi AM, Mei H, Hiepe F, Dorner T (2008) Peripheral B cell abnormalities and disease activity in systemic lupus erythematosus. Lupus 17: 1064-1069.

3. Cheng Lay (2018) The Causes and predictors of mortality in biopsyproven lupus nephritis: the Sarawak experience. Clin Kidney J 11: 56-61.

4. Carter LM, Isenberg DA, Ehrenstein MR (2013) Elevated serum BAFF levels are associated with rising anti-double-stranded DNA antibody levels and disease flare following B cell depletion therapy in systemic lupus erythematosus. Arthritis Rheum 65: 2672-2679.

5. Wallace DJ, Gordon C, Strand V (2013) Efficacy and safety of epratuzumab in patients with moderate/severe flaring systemic lupus erythematosus: results from two randomized, double-blind, placebo-controlled, multicentre studies (ALLEVIATE) and follow up. Rheumatology 52: 1313-1322.

6. Wallace DJ, Kalunian K, Petri MA, Strand V, Houssiau FA, et al. (2014) Efficacy and safety of epratuzumab in patients with moderate/severe active systemic lupus erythematosus: results from EMBLEM, a phase IIb, randomised, double-blind, placebocontrolled, multicentre study. Ann Rheum Dis 73: 183-190.

7. Merrill JT, Neuwelt CM, Wallace DJ, Shanahan JC, Latinis KM, et al. (2010) Efficacy and safety of rituximab in moderately to severely active systemic lupus erythematosus: the randomized, doubleblind, phasell/III systemic lupus erythematosus evaluation of rituximab trial. Arthritis Rheum 62: 222-233.

8. Rovin BH, Furie R, Latinis K, Looney RJ, Fervenza FC, et al. (2012) Efficacy and safety of rituximab in patients with active proliferative lupus nephritis: the lupus nephritis assessment with rituximab study. Arthritis Rheum 64: 1215-1226.

9. Satterthwaite $A B$ (2017) Bruton's tyrosine kinase, a component of $B$ cell signaling pathways, has multiple roles in the pathogenesis of lupus. Front Immunol 8: 1986.

10. Sheau-Pey Wang (2015) Amplification of IL-21 signalling pathway through Bruton's tyrosine kinase in human B cell activation Rheumatology (Oxford) 54: 1488-1497.

11. Moreau T, Calmels B, Barlogis V, Michel G, Tonnelle C, et al. (2007) Potential application of gene therapy to X-linked agammaglobulinemia. Curr Gene Ther 7: 284-294.

12. Walter HS, Rule SA, Dyer MJ, Karlin L, Jones C, et al. (2016) A phase 1 clinical trial of the selective BTK inhibitor ONO/GS-4059 in relapsed and refractory mature B-cell malignancies. Blood 127: 411-419.

13. Walter HS, Jayne S, Rule SA, Cartron G, Morschhauser F, et al. (2017) Long-term follow-up of patients with CLL treated with the selective Bruton's tyrosine kinase inhibitor ONO/GS-4059. Blood. 129: $2808-2810$.

14. Hutcheson J, Vanarsa K, Bashmakov A, Grewal S, Sajitharan D, et al. (2012) Modulating proximal cell signaling by targeting Btk ameliorates humoral autoimmunity and endorgan disease in murine lupus. Arthritis Res Ther 14: R243.

15. Mina-Osorio P, LaStant J, Keirstead N, Whittard T, Ayala J, et al. (2013) Suppression of glomerulonephritis in lupus-prone NZB $x$ NZW mice by RN486, a selective inhibitor of Bruton's tyrosine kinase. Arthritis Rheum 65: 2380-2391.

16. Rankin AL, Seth N, Keegan S, Andreyeva T, Cook TA, et al. (2013) Selective inhibition of BTK prevents murine lupus and antibodymediated glomerulonephritis. J Immunol 191: 4540-4550.

17. Katewa A, Wang Y, Hackney JA, Huang T, Suto E, et al. (2017) Btkspecific inhibition blocks pathogenic plasma cell signatures and myeloid cell-associated damage in IFNalpha-driven lupus nephritis. JCl Insight 2: e90111.

18. Kim YY, Park KT, Jang SY, Lee KH, Byun JY, et al. (2017) HM71224, a selective Bruton's tyrosine kinase inhibitor, attenuates the development of murine lupus. Arthritis Res Ther 19: 211.

19. Chalmers SA, Wen J, Doerner J, Stock A, Cuda CM, et al. (2018) Highly selective inhibition of Bruton's tyrosine kinase attenuates skin and brain disease in murine lupus. Arthritis Res Ther 20: 10.

20. Yasuhiro T, Sawada W, Klein C, Kozaki R, Hotta S, et al. (2017) Antitumor efficacy study of the Bruton's tyrosine kinase (BTK) inhibitor, ONO/GS-4059, in combination with the glycoengineered type II anti-CD20 monoclonal antibody obinutuzumab (GA101) demonstrates superior in vivo efficacy compared to ONO/GS-4059 in combination with rituximab. Leuk Lymphoma 58: 699-707.

21. Du Y, Sanam S, Kate K, Mohan C (2015) Animal models of lupus and lupus nephritis. Curr Pharm Des 21: 2320-2349.

22. Lin W, Seshasayee D, Lee WP, Caplazi P, McVay S, et al. (2015) Dual $B$ cell immunotherapy is superior to individual anti-CD20 depletion or BAFF blockade in murine models of spontaneous or accelerated lupus. Arthritis Rheumatol 67: 215-224.

23. Bekar KW, Owen T, Dunn R, Ichikawa T, Wang W, et al. (2010) Prolonged effects of short-term anti-CD20 B cell depletion therapy in murine systemic lupus erythematosus. Arthritis Rheum 62: 2443-2457.

24. Wang W, Rangel-Moreno J, Owen T, Barnard J, Nevarez S, et al. (2014) Long-term B cell depletion in murine lupus eliminates autoantibody-secreting cells and is associated with alterations in the kidney plasma cell niche. J Immunol 192: 3011-3020.

25. Gensous N, Schmitt N, Richez C, Ueno H, Blanco P (2017) T follicular helper cells, interleukin-21 and systemic lupus erythematosus. Rheumatology (Oxford) 56: 516-523.

26. Hostmann A, Jacobi AM, Mei H, Hiepe F, Dorner T (2008) Peripheral B cell abnormalities and disease activity in systemic lupus erythematosus. Lupus 17: 1064-1069. 